units a program on "The Creation of Patriarchy: Its Implications for Librarianship" (Monday, July 11, 2:00-5:30 p.m.). Gerda Lerner, author of Cre- ation of Patriarchy, will speak on patriarchy. Sharon Hogan will discuss the implications for female academic librarians.

\title{
ACRL meetings in New Orleans
}

\section{A tentative schedule for ALA's New Orleans Conference,}

July 9-14, 1988.

\section{ACRL BOARD OF DIRECTORS}

Orientation breakfast: Saturday, July 9, 8:00-9:00 a.m.

First meeting: Saturday, July 9, 2:00-4:00 p.m.

Second meeting: Tuesday, July 12, 2:00-5:30 p.m.

Executive Committee and Section Officers luncheon: Sunday, July 10, 12:30-2:00 p.m.

Joint meeting with Budget \& Finance and

Planning Committees: Friday, July 8, 4:30-5:30 p.m.

Orientation for new Committee Chairs and new

Section Officers breakfast: Sunday, July 10, 7:30-9:00 a.m.

\section{ACRL GENERAL}

President's Program/Membership: Monday, July 11, 2:00-5:30 p.m.

\section{ACRL DIVISIONAL COMMITTEES}

Academic Library Statistics: Saturday, July 9, 8:00-9:00 a.m.; Monday, July 11, $11: 30$ a.m. $-12: 30$ p.m.

Academic Status: Saturday, July 9, 2:00-4:00 p.m.; Monday, July 11, 9:00-11:00 a.m.; Tuesday, July 12, 9:00-11:00 a.m.

Academic Status-Hearing on Guidelines: Sunday, July 10, 8:00-10:00 p.m.

ACRL/AECT Joint Committee on Two-Year Colleges: Friday, July 8, 9:30 a.m.-12:30 p.m. Audiovisual: Sunday, July 10, 9:00-11:00 a.m. Awards Task Force: Monday, July 11, 9:00-11:00 a.m.
Budget and Finance: Saturday, July 9, 8:00 a.m. $-12: 30$ p.m.; Sunday, July 10, 8:00 a.m.-12:30 p.m., 2:00-5:30 p.m.

Conference Program Planning-Dallas, 1989: Tuesday, July 12, 8:00-9:00 a.m.

Constitution and Bylaws: Saturday, July 9, 8:00-11:00 a.m.; Monday, July 11, 8:00-11:00 a.m.

Continuing Education Courses Advisory: Tuesday, July 12, 9:30 a.m.-12:30 p.m.

Copyright: Saturday, 11:30 a.m.-12:30 p.m.

Copyright-Program: Monday, July 11, 9:30-11:00 a.m.

Extended Campus Library Services Guidelines: Saturday, July 9, 8:00-10:00 p.m.; Sunday, July 10, 8:00-10:00 p.m.; Tuesday, July 12, 9:00-11:00 a.m.

Historically Black College and University Library Project: Tuesday, July 12, 9:00-11:00 a.m.

International Relations Task Force: Tuesday, July 12, 8:00-9:00 a.m.

Legislation: Saturday, July 9, 9:00-11:00 a.m.; Monday, July 11, 2:00-4:00 p.m.

Legislation-Program: Sunday, July 10, 2:00-4:00 p.m.

Librarians as Instructors Task Force: Saturday, July 9, 8:00-9:00 a.m.; Sunday, July 10, 8:00-9:00 a.m.; Monday, July 11, 8:00-9:00 a.m.

Libraries and Computer Centers Task Force: Saturday, July 9, 11:30 a.m.-12:30 p.m.

Library Access Task Force: Saturday, July 9, 11:30 a.m.-12:30 p.m.; Sunday, July 10, 9:00-11:00 a.m.; Tuesday, July 12, 9:00-11:00 a.m. 
Membership: Monday, July 11, 9:30 a.m.-12:30 p.m.

National Conference Executive: Saturday, July 9 , 12:30-2:00 p.m.; Monday, July 11, 8:00-11:00 a.m.

Performance Measures: Saturday, July 9, 9:30 a.m.-12:30 p.m.; Monday, July 11, 9:30 a.m. $-12: 30$ p.m.

Planning: Saturday, July 9, 9:30 a.m.-12:30 p.m.; Monday, July 11, 8:00 a.m.-12:30 p.m.

Professional Association Liaison: Saturday, July 9, 9:00-11:00 a.m.; Sunday, July 10, 2:00-4:00 p.m.

Professional Education: Sunday, July 10, 8:00-11:00 a.m.

Professional Ethics Task Force: Monday, July 11, 8:30-11:00 a.m.

Publications: Sunday, July 10, 2:00-4:00 p.m.; Monday, July 11, 2:00-4:00 p.m.

Research: Sunday, July 10, 9:30 a.m.-12:30 p.m.; Monday, July 11, 9:30 a.m.-12:30 p.m.

Social Responsibility Task Force: Friday, July 8, 8:00-10:00 p.m.

Standards and Accreditation: Saturday, July 9, 8:30 a.m.-12:30 p.m.; Monday, July 11, 8:30 a.m. $-12: 30$ p.m.

\section{ACRL CHAPTERS COUNCIL}

Business: Sunday, July 10, 8:30 a.m.-12:30 p.m.

Strategic Planning Committee: Saturday, July 9, 2:00-4:00 p.m.

\section{ACRL DISCUSSION GROUPS}

Australian Studies: Sunday, July 10, 11:30 a.m. $-12: 30$ p.m.

Black Studies: Sunday, July 10, 2:00-4:00 p.m.

Canadian Studies: Sunday, July 10, 8:00-10:00 p.m.

English and American Literature: Monday, July 11, 8:00-9:00 a.m., 2:00-5:30 p.m.

Extended Campus Library Services: Monday, July 11, 9:00-11:00 a.m.

Fee-Based Information Service Centers in Academic Libraries: Sunday, July 10, 9:30-11:00 a.m.; Monday, July 11, 2:00-4:00 p.m.

Heads of Public/Readers Services: Sunday, July 10, 4:30-5:30 p.m.

Home Economics/Human Ecology Librarians: Sunday, July 10, 4:30-5:30 p.m.

Librarians of Library Science Collections: Saturday, July 9, 9:30-11:00 a.m.

Personnel Administrators and Staff Development Officers: Saturday, July 9, 9:30-11:30 a.m.; Sunday, July 10, 2:00-4:00 p.m.; Tuesday, July 12, 9:30-11:00 a.m.

Public Relations in Academic Libraries: Sunday, July 10, 2:00-5:30 p.m.

Research: Monday, July 11, 9:30-11:00 a.m.

Undergraduate Librarians: Monday, July 11, 9:30 a.m. $-12: 30$ p.m.

\section{ACRL EDITORIAL BOARDS}

Choice: Sunday, July 10, 9:30 a.m.-12:30 p.m.

College \& Research Libraries: Sunday, July 10, 9:00-11:00 a.m.

College \& Research Libraries News: Sunday, July 10, 8:00-9:00 a.m.

Publications in Librarianship: Monday, July 11, 9:30 a.m. $-12: 30$ p.m.

Rare Books \& Manuscripts Librarianship: Saturday, July $9,11: 30$ a.m.-12:30 p.m.

\section{ACRL SECTIONS}

\section{Activity Sections Council}

Meeting: Tuesday, July 12, 11:30 a.m.-12:30 p.m.

\section{Anthropology and Sociology Section}

Program: Monday, July 11, 9:30 a.m.-12:30 p.m.

Tour: Tuesday, July 12, 1:00-5:00 p.m.

Executive: Saturday, July 9, 9:00-11:00 a.m.; Monday, July 11, 2:00-5:00 p.m.

Bibliography: Saturday, July 9, 2:00-4:00 p.m.; Sunday, July 10, 9:00 a.m.-12:30 p.m.

Conference Program Planning-Dallas, 1989: Sunday, July 10, 2:00-4:00 p.m.

Conference Program Planning-Chicago, 1990: Sunday, July 10, 8:00-9:00 a.m.

Nominating: Saturday, July 9, 11:30 a.m.-12:30 p.m.; Monday, July 11, 4:00-5:00 p.m.*

Review and Planning: Monday, July 11, 8:00-9:00 a.m.

\section{Art Section}

Program: Monday, July 11, 9:30 a.m.-12:30 p.m.

Executive: Sunday, July 10, 2:00-4:00 p.m.

Membership: Monday, July 11, 2:00-4:00 p.m.

Conference Program Planning-Dallas, 1989: Sunday, July 10, 9:30-11:00 a.m.

Mission and Goals: Sunday, July 10, 9:30-11:00 a.m.

Publications: Sunday, July 10, 9:30-11:00 a.m.

Technology Sunday, July 10, 9:30-11:00 a.m.

\section{Asian and African Section}

Program: Monday, July 11, 9:30 a.m.-12:30 p.m. Executive: Sunday, July 10, 9:30-11:00 a.m.

\section{Bibliographic Instruction Section}

Program: Sunday, July 10, 2:00-5:30 p.m.

Executive: Friday, July 8, 4:30-5:30 p.m.; Monday, July $11,11: 30$ a.m.-12:30 p.m.; Wednesday, July 13, 8:00-9:00 a.m.

Advisory Council: Saturday, July 9, 8:00-9:00 a.m.; Monday, July 11, 9:00-11:00 a.m.

Clearinghouse: Saturday, July 9, 9:30 a.m.-12:30 p.m.

Communication: Saturday, July 9, 9:30 a.m. $-12: 30$ p.m.

Computer Concerns: Saturday, July 9, 9:30

${ }^{*}$ Meetings with an asterisk are closed meetings. 


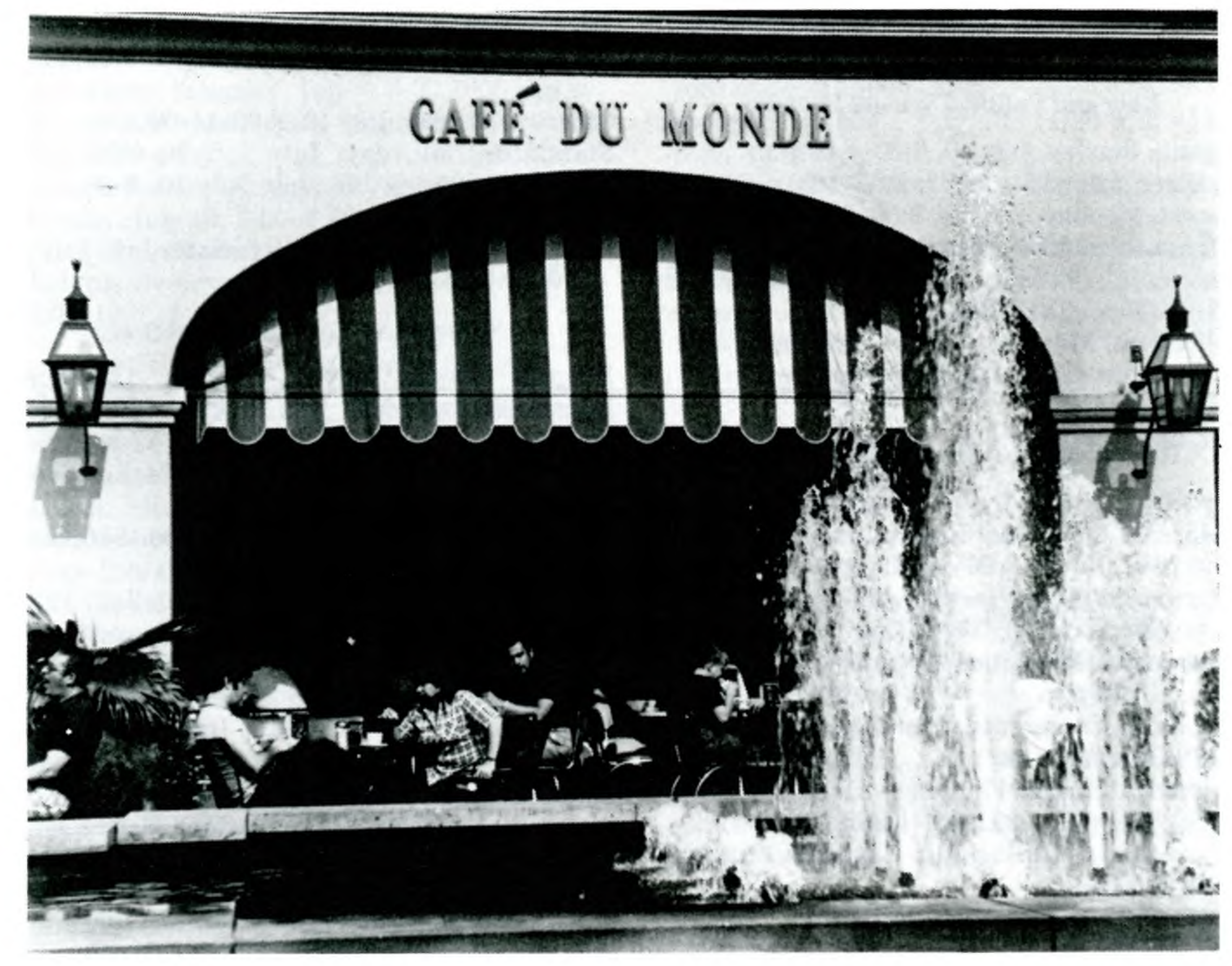

The Café du Monde in New Orleans' French Market is the place to meet for beignets and dark roast café au lait.

a.m.-12:30 p.m.

Conference Program Planning-New Orleans, 1988: Saturday, July 9, 9:30 a.m.-12:30 p.m.

Conference Program Planning-Dallas, 1989: Sunday, July 10, 9:30 a.m.-12:30 p.m.

Continuing Education: Saturday, July 9, 9:30 a.m. $-12: 30$ p.m.

Education for Bibliographic Instruction: Sunday, July 10, 9:30 a.m.-12:30 p.m.

Miriam Dudley Award: Saturday, July 9, 11:30 a.m. $-12: 30$ p.m.*

Nominating: Saturday, July 9, 11:30 a.m.-12:30 p.m.*

Planning: Saturday, July 9, 9:30 a.m.-12:30 p.m.

Policy: Sunday, July 10, 9:30 a.m.-12:30 p.m.

Research: Saturday, July 9, 9:30 a.m.-12:30 p.m.

\section{College Libraries Section}

Program: Sunday, July 10, 9:30 a.m.-12:30 p.m.

Executive: Saturday, July 9, 11:30 a.m.-12:30 p.m.; Tuesday, July 12, 11:30 a.m.-12:30 p.m. CLIP Notes: Monday, July 11, 9:00-11:00 a.m.

College Library Directors: Sunday, July 10, 2:00-4:00 p.m.

Historical Commission: Monday, July 11, 8:00 a.m. $-12: 30$ p.m.

National Advisory Council Steering: Saturday, July 9, 2:00-4:00 p.m.; Monday, July 11,
9:00-11:00 a.m.

Newsletter: Saturday, July 9, 2:00-4:00 p.m.

Real Income: Saturday, July 9, 2:00-4:00 p.m.

Standards: Saturday, July 9, 2:00-4:00 p.m.;

Tuesday, July 12, 9:00-11:00 a.m.

Community and Junior College Libraries Section

Program: Saturday, July 9, 9:30 a.m.-12:30 p.m.

Executive: Wednesday, July 13, 2:00-4:00 p.m.

Committees: Sunday, July 10, 9:00-11:00 a.m.

Education and Behavioral Sciences Section

Program: Monday, July 11, 9:30 a.m.-12:30 p.m.

Executive: Saturday, July 9, 7:00-8:00 a.m.; Tuesday, July 12, 8:00-11:00 a.m.

Bibliographic Instruction for Educators: Sunday, July 10, 9:00 a.m. $-12: 30$ p.m.

Conference Program Planning-New Orleans, 1988: Saturday, July 9, 8:00-9:00 a.m.

Conference Program Planning-Dallas, 1989: Sunday, July 10, 2:00-4:00 p.m.; Monday, July 11, 2:00-4:00 p.m.

Curriculum Materials: Sunday, July 10, 2:00-5:30 p.m.

Education Librarian of the Year: Saturday, July 9, 9:30-11:00 a.m.

Psychology/Psychiatry: Monday, July 11, 2:00-5:30 p.m. 
Publications: Monday, July 11, 8:00-9:00 a.m.

Section Review: Saturday, July 9, 2:00-4:00 p.m.

\section{Law and Political Science Section}

Program: Sunday, July 10, 2:00-4:00 p.m.

Executive: Saturday, July 9, 2:00-4:00 p.m.

Business: Monday, July 11, 9:00-11:00 a.m.

Conference Program Planning-Dallas, 1989: Saturday, July 9, 9:00-11:00 a.m.; Monday, July 11, 11:30 a.m. $-12: 30$ p.m.

Publications: Monday, July 11, 8:00-9:00 a.m.

Section Review and Planning: Monday, July 11, 8:00-9:00 a.m.

\section{Rare Books and Manuscripts Section}

Program: Sunday, July 10, 2:00-5:30 p.m.

RBMS/ALTA/LAMA Program on Inside Theft: Saturday, July 9, 8:00-10:00 p.m.

Executive: Sunday, July 10, 11:30 a.m.-12:30 p.m.; Monday, July 11, 9:30 a.m.-12:30 p.m.

Borrowing for Exhibition: Saturday, July 9, 11:30 a.m. $-12: 30$ p.m.

Cambridge Conference Planning: Sunday, July 10, 9:00-11:00 a.m.

Conference Program Planning-Dallas, 1989: Saturday, July 9 , 11:30 a.m.-12:30 p.m.

Conservators' Collations: Saturday, July 9, 2:00-4:00 p.m.

Continuing Education: Saturday, July 9, 2:00-4:00 p.m.

Curators and Conservators: Saturday, July 9, 11:30 a.m. $-12: 30$ p.m.

Ethical Standards Review: Saturday, July 9, 9:00-11:00 a.m.

Exhibition Catalogues Award: Saturday, July 9, 9:00-11:00 a.m.

Gifts and Appraisals: Saturday, July 9, 9:00-11:00 a.m.

Guidelines on Archives and Manuscripts: Saturday, July 9, 2:00-4:00 p.m.

Information Exchange: Sunday, July 10, 8:00-10:00 p.m.

Legislative Information: Saturday, July 9, 2:00-4:00 p.m.

Literary Rights: Sunday, July 10, 9:00-11:00 a.m.

Manuscripts and Other Formats: Saturday, July 9, 9:00-11:00 a.m.

MARC for Special Collections: Saturday, July 9, 11:30 a.m.-12:30 p.m.

Orientation Manual: Saturday, July 9, 11:30 a.m. $-12: 30$ p.m.

\section{University Library Standards draft}

The June issue of C $\triangleleft R L$ News will have the most recent draft of the "Standards for University Libraries," currently under revision by the University Libraries Section. Hearings will be held in New Orleans on July 10, 1988, 8:00-10:00 p.m.
Publications: Sunday, July 10, 9:00-11:00 a.m.

Records Retention: Saturday, July 9, 9:00-11:00 a.m.

Security: Sunday, July 10, 9:00-11:00 a.m.

Standards: Saturday, July 9, 2:00-4:00 p.m., 8:00-10:00 p.m.; Sunday, July 10, 9:00-11:00 a.m.

Statistical Survey Feasibility: Saturday, July 9, 2:00-4:00 p.m.

\section{Science and Technology Section}

Program: Tuesday, July 12, 9:30 a.m.-12:30 p.m.

Executive: Saturday, July 9, 9:00-11:00 a.m.; Tuesday, July 12, 2:00-4:00 p.m.

Comparison of Science and Engineering Libraries: Monday, July 11, 2:00-4:00 p.m.

Conference Proceedings Task Force: Saturday, July 9, 9:00-11:00 a.m.

Conference Program Planning-Dallas, 1989: Monday, July 11, 8:30 a.m.-12:30 p.m.

Conference Program Planning-New Orleans, 1988: Sunday, July 10, 8:30 a.m.-12:30 p.m.

Discussion Group: Saturday, July 9, 2:00-4:00 p.m.

Legislation: Sunday, July 10, 9:00-11:00 a.m.

Membership: Sunday, July 10, 9:00-11:00 a.m.

Newsletter: Monday, July 11, 9:00-11:00 a.m.

Nominating: Monday, July 11, 9:00-11:00 a.m.*

Oberly Award: Sunday, July 10, 9:00-11:00 a.m.*

Planning: Monday, July 11, 9:00-11:00 a.m.

Publisher Concerns: Sunday, July 10, 9:30-12:30 p.m.

Science Databases: Monday, July 11, 2:00-4:00 p.m.

Subject and Bibliographic Access: Monday, July 11, 2:00-5:30 p.m.

\section{Slavic and East European Studies Section}

Program: Sunday, July 10, 2:00-4:00 p.m.

Executive: Monday, July 11, 2:00-4:00 p.m.

Conference Program Planning-Dallas, 1989: Sunday, July 10, 11:30 a.m.-12:30 p.m.

Continuing Education: Sunday, July 10, 9:30-11:00 a.m.

Cyrillic Romanization and Automation: Monday, July 11, 9:00-11:00 a.m.

Membership: Sunday, July 10, 4:30-5:30 p.m.

Newsletter: Monday, July 11, 11:30 a.m.-12:30 p.m.

Nominating: Sunday, July 10, 8:00-9:00 a.m.*

\section{University Libraries Section}

Program: Saturday, July 9, 2:00-4:00 p.m.

Steering: Saturday, July 9, 9:00-11:00 a.m.; Monday, July 11, 9:30-11:00 a.m.

Conference Program Planning-Dallas, 1989: Sunday, July 10, 9:00-11:00 a.m.

Conference Program Planning-New Orleans, 1988: Saturday, July 9, 8:00-9:00 a.m.

Current Topics Planning: Sunday, July 10, 9:00-11:00 a.m., 2:00-4:00 p.m.

Guidelines for Branch Libraries: Saturday, July 9 , 
2:00-4:00 p.m.; Tuesday, July 12, 2:00-4:00 p.m.

Nominations: Saturday, July 9, 8:00-9:00 a.m. ${ }^{*}$

Performance Measures: Sunday, July 10, 2:00-4:00 p.m.

Standards Review: Friday, July 8, 1:00-4:00 p.m.; Sunday, July 10, 2:00-4:00 p.m.; Monday, July 11, 2:00-4:00 p.m.

Standards Review-Hearings: Sunday, July 10, 8:00-10:00 p.m.

\section{Western European Specialists Section}

Program: Monday, July 11, 9:30 a.m.-12:30 p.m.

Executive: Saturday, July 9, 2:00-4:00 p.m.; Tuesday, July 12, 2:00-4:00 p.m.

Classical, Medieval, Renaissance: Saturday, July 9, 2:00-4:00 p.m.

College Libraries and European Studies: Sunday, July 10, 4:30-5:30 p.m.

Conference Program Planning-Dallas, 1989: Sunday, July 10, 9:30-11:00 a.m.

Continuing Education: Tuesday, July 12, 8:00-9:00 a.m.

Directory: Tuesday, July 12, 11:30 a.m.-12:30 p.m.

European Conference: Tuesday, July 12, 8:00-9:00 a.m.
Germanists: Sunday, July 10, 8:00-10:00 p.m.

Membership/General Discussion Group: Monday, July 11, 2:00-4:00 p.m.

Newsletter: Sunday, July 10, 11:30 a.m.-12:30 p.m.

Nijhoff Study Grant Jury: Sunday, July 10, 9:30-11:00 a.m.*

Nominating: Sunday, July 10, 8:00-9:00 a.m.*

Publications: Tuesday, July 12, 9:30-11:00 a.m.

Research and Planning: Sunday, July 10, 2:00-4:00 p.m.

\section{Women's Studies Section}

Program: Saturday, July 9, 9:30 a.m.-12:30 p.m.

Executive: Friday, July 8, 2:00-4:00 p.m.

Membership: Monday, July 11, 9:30-11:00 a.m.

Communications: Monday, July 11, 11:30 a.m. $-12: 30$ p.m.

Conference Program Planning-Dallas, 1989:

Monday, July 11, 11:30 a.m.-12:30 p.m.

Conspectus: Saturday, July 9, 8:00-10:00 p.m.

National Conference: Monday, July 11, 11:30 a.m. $-12: 30$ p.m.

Nominating: Monday, July 11, 11:30 a.m.-12:30 p.m.*

Technical Services: Monday, July 11, 11:30 a.m. $-12: 30$ p.m.

\section{Hearings on academic status}

\section{and certification}

\section{Guidelines to be reconsidered at Annual Conference in New Orleans on July 10.}

\footnotetext{
A

the American Library Association Conference this July, the ACRL Academic Status Committee will be sponsoring a hearing on two new statements. In order for you to have time to read and think about them, they are printed here in their entirety.
}

The membership of the Association of College and Research Libraries adopted on June 26, 1971, "Standards for Faculty Status for College and University Librarians." However, many college and university librarians do not hold faculty status; instead they have academic status. These librarians 